\title{
Willingness of patients to change surgeons for a shorter waiting time for joint arthroplasty
}

\author{
Barbara Conner-Spady PhD, Claudia Sanmartin PhD, Geoffrey Johnston MD MBA, \\ John McGurran MSc, Melissa Kehler MA, Tom Noseworthy MD MPH
}

\section{ABSTRACT}

Background: To improve access to care, many jurisdictions have proposed waiting-time benchmarks and guarantees. We assessed the willingness of patients to consider changing their surgeon to one with a shorter waiting time for arthroplasty.

Methods: We mailed a questionnaire to 2 random samples of patients who either were awaiting hip or knee replacement arthroplasty or had had one of these procedures within the preceding 3-12 months. We used logistic regression to assess the determinants of patients' likelihood to consider changing surgeons.

Results: Of 1200 responses from a sample of 2000, 557 (46\%) were from patients awaiting surgery and 643 (54\%) were from people who had undergone surgery. The mean age of respondents was 69.9 years (standard deviation 10.8), and $682(57 \%)$ were women. The median waiting time for surgery was 8 months. Overall, $753(63 \%)$ of the patients were unlikely to consider changing surgeons. Increased likelihood of changing surgeons was associated with male sex (adjusted odds ratio [OR] 1.49, 95\% confidence interval [CI] 1.10-2.02), a high school education or higher (OR 1.73, 95\% Cl 1.152.62) and having already undergone surgery (OR 1.71, 95\% CI 1.19-2.46). Decreased likelihood was associated with preference for a particular surgeon before referral (OR 0.57, 95\% Cl 0.42-0.79), a better score on the EuroQol (EQ-5D) index (a measure of health-related quality of life) (OR $0.39,95 \% \mathrm{Cl}$ $0.24-0.66)$, perception that the waiting time to see the surgeon was acceptable (OR $0.50,95 \% \mathrm{Cl} 0.36-0.70$ ), perception that the waiting time to surgery was acceptable (OR 0.62 , $95 \% \mathrm{Cl} 0.43-0.91$ ) and perceived fairness of treatment (OR $0.53,95 \% \mathrm{Cl} 0.36-0.78$ ).

Interpretation: Despite long waits for surgery, most patients, if given the choice, would be unlikely to change their surgeon to one with a shorter waiting time.

Une version française de ce résumé est disponible à l'adresse www.cmaj.ca/cgi/content/full/179/4/327/DC1

CMAJ 2008;179(4):327-32

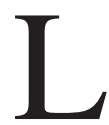

ong waiting times for elective surgery are a concern in countries with publicly funded health care systems. ${ }^{1}$ To try to improve access, governments in Canada and some other countries in the Organisation for Economic Co-operation and Development have proposed or implemented waiting-time benchmarks and care guarantees. ${ }^{2}$ These benchmarks, typically between 3 and 12 months, usually refer to the time between assessment by a specialist and in-patient treatment. ${ }^{3}$

Waiting times for hip and knee arthroplasty are perceived as excessive in many countries, including Canada. ${ }^{4-6}$ In 2005, Canada's federal, provincial and territorial health ministers set a target waiting time of 26 weeks for hip and knee arthroplasty, and in 2007 they agreed to establish waiting-time guarantees by 2010 in selected priority areas, including joint arthroplasty. ${ }^{7}$ A waiting-time guarantee implies that, if necessary medical treatment for a publicly insured service is not available within a medically acceptable timeframe, patients may receive treatment at another facility, even outside their home province, at public expense. This change in the location of treatment implies that another surgeon would perform the surgery. However, patients are not always willing to accept re-referral to a provider with a shorter waiting time..$^{8-12}$

Most other studies investigating patient choice in the context of long waiting times have referred to the choice of an alternative hospital rather than the choice of an alternative surgeon. Even though many patients may indicate that they are willing to travel to another hospital, fewer actually choose such a change. ${ }^{11}$ In addition to waiting time, ${ }^{8,13}$ other factors influencing provider choice are the hospital's reputation, follow-up care and travel time..$^{8,13,14}$ Little is known about what factors patients consider and what information they want and can use when choosing a provider. ${ }^{11,15}$

We sought to assess patients' willingness to consider changing to a surgeon with a shorter waiting time for hip and knee arthroplasty. We formulated the following research questions: Would patients consider changing to a surgeon with shorter waiting times? What factors influence patients' willingness to consider changing surgeons? Do patients waiting for surgery have a different perspective than patients who have already undergone their surgery?

From the Department of Community Health Sciences (Conner-Spady, Sanmartin, Noseworthy), University of Calgary, Calgary, Alta.; the Western Canada Waiting List Project (Conner-Spady, McGurran, Noseworthy), Calgary, Alta.; Statistics Canada (Sanmartin), Ottawa, Ont.; the Department of Surgery (Orthopaedics) (Johnston), College of Medicine, University of Saskatchewan, Saskatoon, Sask.; the Department of Public Health Sciences (McGurran), University of Toronto, Toronto, Ont.; and the Department of Psychology, University of Regina (Kehler), Regina, Sask. 


\section{Methods}

\section{Study population}

In Saskatchewan, all joint arthroplasty procedures are performed in 4 health regions, of which 2 (Saskatoon and Regina Qu'Appelle) account for about $86 \%$ of the surgeries. As part of a recently implemented patient assessment process to improve access to surgical services ${ }^{16}$ orthopedic surgeons assign priority scores to patients who are waiting for surgery according to the urgency of their condition. ${ }^{17,18}$ At the time of booking, usually within a few days of the decision date for surgery, patients are listed on the Surgical Patient Registry, a data repository that tracks all patients waiting for surgery. Typically, patients learn the date of surgery a few weeks before the procedure.

For this study, Saskatchewan Health selected 2 groups of patients 18 years and older from the Surgical Patient Registry; selection involved random sampling, stratified by group and by joint. The first group consisted of 1000 patients on a waiting list for hip or knee arthroplasty; the second group, also numbering 1000, had undergone hip or knee arthroplasty 3-12 months previously. We based the sample size on an estimated response rate of $60 \%$; this sample size was sufficient to minimize sampling error with a minimum of 50 cases per independent variable. ${ }^{19}$

\section{Data collection}

For this cross-sectional study, we mailed a questionnaire in November 2006 to the 1000 patients in each group. We sent 2 reminder letters (each with a copy of the questionnaire and a self-addressed stamped envelope) to nonrespondents 6 and 12 weeks after the initial mailing. We did not send follow-up correspondence to patients in the group scheduled for surgery if they had either undergone or cancelled their surgery since the initial mailing. We asked patients for consent to link their questionnaire data to data in the Surgical Patient Registry (specifically waiting time and rural or urban residence). The research ethics boards of the University of Calgary, the University of Saskatchewan and the Regina Qu'Appelle Health Region provided ethics approval for the study.

Using the questionnaire, we asked both groups about their experiences during the waiting period. We based the questionnaire items on our research questions and a literature review. We had previously tested most of the items in a consecutive postoperative sample of patients who had undergone hip and knee arthroplasty. ${ }^{20}$ In addition, for the current study we convened 4 focus groups with a total of 43 participants, 2 groups of participants awaiting surgery and 2 groups who had undergone surgery, who were randomly selected from the Surgical Patient Registry and stratified by community (Saskatoon or Regina), study group and joint. The focus groups reviewed the survey questions for clarity and interpretation and assessed whether important issues were covered in the questionnaire.

We used the following question to determine patients' willingness to change surgeons: "If you had a choice of going to another equally qualified orthopedic surgeon with a shorter waiting time, how likely would you be to consider changing

\section{Box 1: Questionnaire items*}

- If you had a choice of going to another equally qualified orthopedic surgeon with a shorter waiting time, how likely would you be to consider changing surgeons? + (4-point scale, from "very likely" to "not at all likely")

- Did your family doctor provide you with any option as to which orthopedic surgeon you might see? (yes/no)

- Did you have a preference for an orthopedic surgeon before your family doctor referred you to one? (yes/no)

- How acceptable is the length of time that you have been waiting for your surgery? (defined as between when you and your surgeon decided to go ahead with the surgery and now) + (4-point scale, from "very acceptable" to "very unacceptable")

- How acceptable was the waiting time between when you and your family doctor decided that you should see an orthopedic surgeon and when you actually visited the surgeon?+ (4-point scale, from "very acceptable" to "very unacceptable")

- How fairly do you feel you are being treated in regards to the length of time that you are waiting for your joint replacement surgery?+ (5-point scale, from "very fairly" to "very unfairly")

*For the postsurgery group, the verb tense for the questions was changed as necessary. For example, the sixth question read "How fairly did you feel you were treated in regards to the length of time that you waited for your most recent joint replacement surgery?"

tResponses for these 4 items were collapsed into 2 categories for the logistic regression model.

surgeons?" The questionnaire also included 2 open-ended questions exploring the reasons for the participants' decision, but we have not reported responses to those questions here.

We assessed health-related quality of life with the EuroQol (EQ-5D) index and EQ visual analogue scale, ${ }^{21}$ standardized generic measures that have been tested in patients who have undergone arthroplasty. ${ }^{22}$ For the group that had already undergone surgery, we calculated waiting time from the initial booking date to the surgery date; for the group that was still waiting for surgery, we calculated waiting time from the initial booking date to the date when the survey was returned. We omitted time for any patient-initiated delays (i.e., if surgery was postponed for personal reasons).

\section{Data analysis}

We used logistic regression to build a model of factors explaining patients' likelihood to change to a surgeon with a shorter waiting time (likely v. unlikely). ${ }^{23}$ We used $\chi^{2}$ analysis and logistic regression to test the univariable association between potential explanatory variables and the dependent variable. We used $p$ values less than 0.25 for initial selection of variables to be entered into the multiple logistic regression model, ${ }^{23}$ and we retained significant variables $(p<0.05)$ in the final model. We analyzed the following demographic variables: age, sex, marital status (married or with a partner v. other), education (high school completion or higher v. less than high school completion), living arrangement (living alone v. living with others) and place of residence (rural v. urban). We also screened for several other potentially ex- 
planatory variables: joint (hip or knee), first or second arthroplasty procedure, group (waiting or postsurgery), waiting time, option of a surgeon before referral (yes or no), preference for a surgeon before referral (yes or no), the EQ-5D index, perceived acceptability of the wait for surgery and of the wait to see the surgeon (yes or no) and perceived fairness with regard to the length of the wait (fair v. unfair or neutral) (Box 1).

\section{Results}

Of the 2000 questionnaires distributed, 1330 (611 from patients waiting for surgery and 719 from patients who had undergone surgery) were returned (Figure 1). There were no significant differences in sex, joint replaced (or to be replaced) or waiting time between respondents and nonrespondents. Respondents were slightly older than nonrespondents (69.5 v. 66.4 years, $p<0.001)$. Of the 1330 respondents, 1200 patients (557 who were waiting for surgery and 643 who had undergone surgery) had completed the question about surgeon choice (response rate $66 \%$ of eligible participants; see Figure 1) and could therefore be included in the analysis. The mean age of these 1200 respondents was 69.9 years (standard deviation 10.8, range 24-97 years), and 682 (57\%) were female (Table 1). Overall, 1009 respondents $(84 \%)$ consented to have their survey data linked with data in the Surgical Patient Registry. We included patients with waiting-time registry data in the logistic regression model.

Patients waited a median of 8 months for surgery (Table 1). Those who had already undergone surgery were significantly more accepting of the wait and were more likely to feel that they had been treated fairly with regard to the waiting time. Thirty-seven percent of the patients (424/1161) had a preference for a particular orthopedic surgeon before referral, and $42 \%$ $(478 / 1139)$ reported that the family doctor had provided options as to the choice of surgeon. Of those with a preference for a particular surgeon, 35\% (147/424) reported having undergone arthroplasty previously. Three hundred and eighty-nine (32\%) of the participants were not very likely and 364 (30\%) were not at all likely to consider changing surgeons (Table 2). In the univariable analysis, variables significantly associated with a greater likelihood of changing surgeons were lower age, male sex, first surgery, no prior surgeon preference, worse EQ-5D index score, perception that the waiting time to see the surgeon and waiting time to surgery were unacceptable, and perception of not being treated fairly.

In addition to the significant variables determined by logistic regression, we retained age and waiting time in the final model because of their clinical relevance (Figure 2). After adjustment for other variables in the model, the variables significantly associated with a greater likelihood of changing surgeons were male sex, at least high school completion and group (postsurgery). Preference for a particular surgeon before referral, better EQ-5D index, perception that the waiting time to see the surgeon was acceptable, perception that the waiting time to surgery was acceptable and perception of fair treatment with regard to waiting time were significantly associated with being less likely to change surgeons.

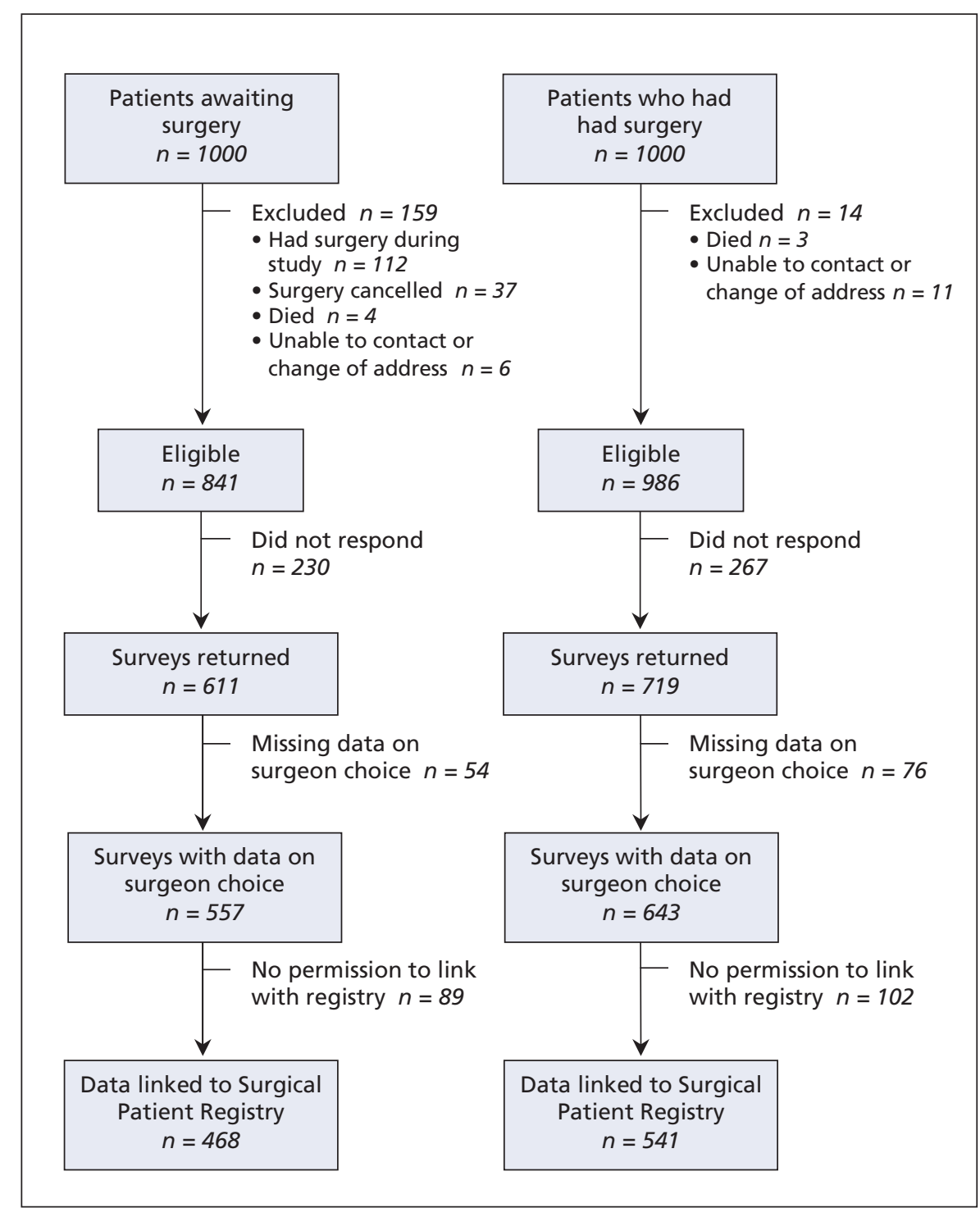

Figure 1: Flow diagram showing selection of patients to receive survey about willingness to change surgeons, return of surveys, and identification of surveys with sufficient data for analysis. 
Table 1: Characteristics of patients who were waiting for or who had undergone hip or knee arthroplasty

\begin{tabular}{|c|c|c|c|}
\hline \multirow[b]{2}{*}{ Characteristic } & \multicolumn{2}{|c|}{ No. $(\%)$ of patients* } & \multirow[b]{2}{*}{$p$ valuet } \\
\hline & $\begin{array}{l}\text { Waiting for surgery } \\
\qquad n=557\end{array}$ & $\begin{array}{l}\text { After surgery } \\
\quad n=643\end{array}$ & \\
\hline Age, yr, mean (SD) & $69.2(10.9)$ & $70.5(10.7)$ & 0.054 \\
\hline Female & $305 / 557(55)$ & $377 / 643(59)$ & 0.17 \\
\hline Married or with partner & $369 / 540(68)$ & $436 / 632(69)$ & 0.81 \\
\hline Living alone & $130 / 538(24)$ & $165 / 631(26)$ & 0.44 \\
\hline Education (completed high school or higher) & $415 / 528(79)$ & $481 / 620(78)$ & 0.68 \\
\hline Urban residence & $245 / 468(52)$ & 267/541 (49) & 0.34 \\
\hline First surgery & $390 / 557(70)$ & $424 / 643(66)$ & 0.14 \\
\hline Hip replacement & $288 / 557(52)$ & $323 / 643(50)$ & 0.63 \\
\hline EuroQol (EQ-5D) index, $\neq$ mean (SD) & $0.34(0.35)$ & $0.67(0.28)$ & $<0.001$ \\
\hline EQ visual analogue scale, § mean (SD) & $56.50(19.6)$ & $71.87(16.68)$ & $<0.001$ \\
\hline Waiting time, mo, mean (SD) & $10.0(7.5)$ & $10.4(8.3)$ & 0.33 \\
\hline Waiting time, mo, median (IQR) & $8.0(4.6-13.4)$ & $8.3(3.7-15.5)$ & \\
\hline Waiting time to see surgeon acceptable & $340 / 522(65)$ & 428/599 (71) & 0.023 \\
\hline Waiting time to surgery acceptable & 238/536 (44) & $383 / 628(61)$ & $<0.001$ \\
\hline Treated fairly with regard to waiting time & $202 / 530(38)$ & $420 / 622(68)$ & $<0.001$ \\
\hline Had a preference for surgeon & 194/538 (36) & 230/623 (37) & 0.76 \\
\hline Given option of a surgeon & $224 / 531(42)$ & $254 / 608(42)$ & 0.89 \\
\hline
\end{tabular}

Note: IQR = interquartile range, SD = standard deviation.

*Unless stated otherwise.

tBased on $\chi^{2}$ test for categorical variables or $t$ test for continuous variables.

¥The EQ-5D index has values from -0.59 (health state worse than death) to 1.00 (full health).

$\S$ The EQ visual analogue scale has values from 0 (worst imaginable health state) to 100 (best imaginable health state).

\section{Interpretation}

In this study, we examined factors affecting patients' perspectives about changing their surgeon to an equally qualified one with a shorter wait, if given the choice. The response rate was acceptable, the sample was randomly chosen, and there were marginal differences in age between respondents and nonrespondents.

Views on acceptability of waiting time, fairness and willingness to change surgeons among patients who had already undergone surgery could be influenced by recall bias and by the removal of uncertainty about how long they would wait. The mean EQ-5D index score for each group was comparable to scores for other similar populations. ${ }^{5,24}$ Even though patients waited a median of 8 months, $63 \%$ of the patients reported that they would be unlikely to consider changing surgeons. Once patients have developed trust in their surgeons, they are willing to wait unless their pain becomes unbearable (results not reported). Other reasons that patients gave for their decision were the surgeon's reputation, personal experience with the surgeon, recommendations from friends and family, the family doctor's recommendation and location. Similar to our findings, $30 \%$ of patients waiting for elective procedures in the United Kingdom would always choose their home hospital over one with a shorter wait, although men were more likely than women to select faster treatment at an alternative hospital. ${ }^{13}$ In Denmark, however, $60 \%$ of patients waiting more than 6 months for joint surgery accepted re-referral to another hospital with a shorter wait. ${ }^{8}$ Waiting time, distance, travel time and previous experience with the hospital influenced their acceptance of re-referral.

Many countries have introduced choice of an alternative provider as a way to increase access to care, ${ }^{3,9,25}$ but success

Table 2: Likelihood that patient would change to a surgeon with a shorter waiting time*

\begin{tabular}{lccc}
\hline & \multicolumn{3}{c}{ No. (\%) of patients } \\
\cline { 2 - 4 } \multicolumn{1}{l}{ Likelihood } & $\begin{array}{c}\text { Waiting } \\
n=557\end{array}$ & $\begin{array}{c}\text { After surgery } \\
n=643\end{array}$ & $\begin{array}{c}\text { Overall } \\
n=1200\end{array}$ \\
\hline Very likely & $128(23)$ & $136(21)$ & $264(22)$ \\
$\begin{array}{l}\text { Somewhat } \\
\text { likely }\end{array}$ & $94(17)$ & $89(14)$ & $183(15)$ \\
Not very likely & $198(36)$ & $191(30)$ & $389(32)$ \\
Not at all likely & $137(25)$ & $227(35)$ & $364(30)$ \\
\hline
\end{tabular}

${ }^{*} \chi^{2}$ analysis for group (waiting for surgery or after surgery) v. likelihood (likely or unlikely), $p=0.08$ 
Variable

Acceptable waiting time

to see surgeon

Acceptable waiting time to surgery

Age

EuroQol (EQ-5D) index

Education

Perceived fairness

regarding waiting time

Group

Sex

Surgeon preference

Waiting time
Likelihood of

changing surgeon

$\longleftarrow$ Decreased Increased $\longrightarrow$

OR $(95 \% \mathrm{CI})$

$0.50(0.36-0.70)$

$0.62(0.43-0.91)$

$0.98(0.97-1.00)$

$0.39(0.24-0.66)$

$1.73(1.15-2.62)$

$0.53(0.36-0.78)$

$1.71(1.19-2.46)$

$1.49(1.10-2.02)$

$0.57(0.42-0.79)$

$1.00(0.98-1.02)$

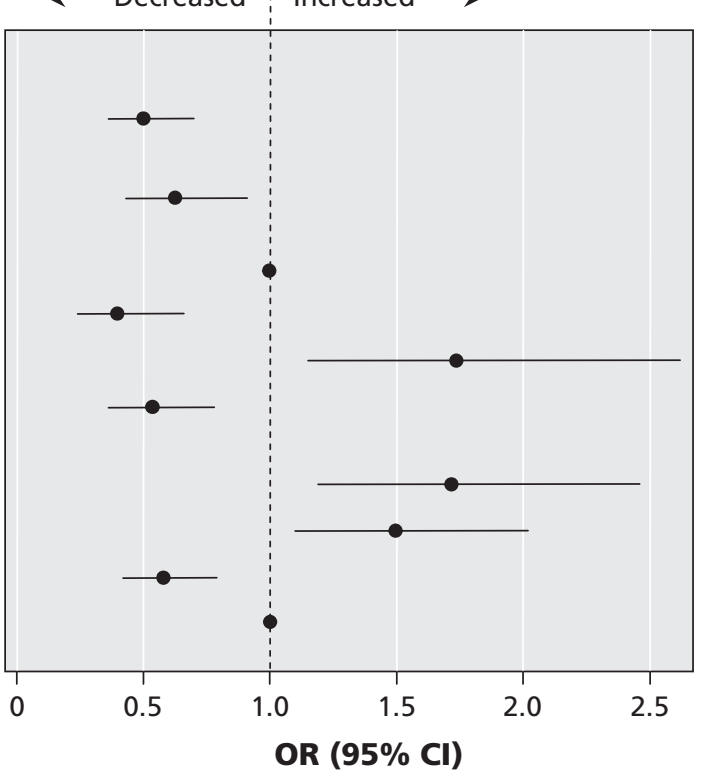

Figure 2: Adjusted odds ratios for logistic regression model of the determinants of patients' likelihood of changing surgeons to one with a shorter waiting time for joint arthroplasty. $\mathrm{OR}=$ odds ratio, $\mathrm{Cl}=$ confidence interval.

has been variable, possibly because of a lack of knowledge about what information patients want and can use in choosing a provider. ${ }^{15}$ Having sufficient information to make an informed choice is important $;^{26}$ however, the extent to which patients use available information may be limited. ${ }^{25,27,28}$ Some patients want their physicians to make this type of decision, and others want more information about health care service quality and outcomes so that they can make meaningful decisions in choosing a provider. ${ }^{15}$

In Saskatchewan, despite long waits for surgery, we found that most patients were unlikely to change to a surgeon with a shorter wait. It is unclear whether these results will be generalizable to other provinces and countries with different characteristics such as waiting time, population density, climate and travel distance. If waiting-time care guarantees are introduced with sufficient additional resources, priority systems and other management policies, they are more likely to fulfill their intended purpose. ${ }^{3}$ An evaluation of these policies is essential to assess the effects of implementation on access to care. Given the recent announcement that waiting-time guarantees will be implemented for selected scheduled procedures, an understanding of the issues that patients consider when deciding to accept an alternative provider is valuable for health care policy-makers. ${ }^{11}$ The results from our study provide a basis for more extensive study of the issues that are relevant in patients' choices in the context of waiting-time guarantees.

\section{This article has been peer reviewed.}

Competing interests: None declared.
Contributors: Barbara Conner-Spady contributed to the conception and design of the study and to the analysis and interpretation of the data; she also drafted the article. Claudia Sanmartin contributed to the conception and design of the study and to the analysis of the data. Geoffrey Johnston and John McGurran contributed to the study design. Melissa Kehler contributed to the acquisition of data. Tom Noseworthy contributed to the conception and design of the study. All of the authors reviewed the manuscript for intellectual content and gave final approval.

Acknowledgement: We acknowledge Saskatchewan Health for its support and assistance.

Funding: This study was funded by the Canadian Institutes of Health Research (grant no. PPG-74829).

\section{REFERENCES}

1. Access to health care services in Canada. Ottawa (ON): Statistics Canada; 2006.

2. Hanning M, Spangberg UW. Maximum waiting time - A threat to clinical freedom? Implementation of a policy to reduce waiting times. Health Policy 2000;52:15-32.

3. Siciliani L, Hurst J. Tackling excessive waiting times for elective surgery: a comparative analysis of policies in 12 OECD countries. Health Policy 2005;72:201-15.

4. Snider MG, MacDonald SJ, Pototschnik R. Waiting times and patient perspectives for total hip and knee arthroplasty in rural and urban Ontario. Can J Surg 2005;48: 355-60.

5. Ostendorf M, Buskens E, van Stel H, et al. Waiting for total hip arthroplasty: avoidable loss in quality time and preventable deterioration. J Arthroplasty 2004; 19:302-9.

6. Fielden JM, Cumming JM, Horne JG, et al. Waiting for hip arthroplasty: economic costs and health outcomes. J Arthroplasty 2005;20:990-7.

7. Canada's new government announces patient wait times guarantees with all the provinces and territories [media release]. Ottawa $(\mathrm{ON})$ : Government of Canada, Canada News Centre; 2007. Available: http://news.gc.ca/web/view/en/index.jsp ?articleid=290189\&keyword=Patient+Wait+Times+Guarantees $+\&$ (accessed 2008 May 8).

8. Birk HO, Henriksen LO. Why do not all hip and knee patients facing long waiting times accept re-referral to hospitals with short waiting time? Questionnaire study. Health Policy 2006;77:318-25.

9. Thomson S, Dixon A. Choices in health care: the European experience. J Health Serv Res Policy 2006;11:167-71. 
10. Howell GP, Richardson D, Forester A, et al. Long distance travel for routine elective surgery: questionnaire survey of patients' attitudes. BMJ 1990;300:1171-3.

11. Brouwer W, van Exel J, Hermans B, et al. Should I stay or should I go? Waiting lists and cross-border care in the Netherlands. Health Policy 2003;63:289-98.

12. Dawson D, Jacobs R, Martin S, et al. Is patient choice an effective mechanism to reduce waiting times? Appl Health Econ Health Policy 2004;3:195-203.

13. Burge P, Devlin N, Appleby J, et al. Do patients always prefer quicker treatment? A discrete choice analysis of patients' stated preferences in the London patient choice project. Appl Health Econ Health Policy 2004;3:183-94.

14. Fotaki M. The impact of market oriented reforms on choice and information: a case study of cataract surgery in outer London and Stockholm. Soc Sci Med 1999; 48:1415-32.

15. Ovretveit J. Informed choice? Health service quality and outcome information for patients. Health Policy 1996;37:75-90.

16. Glynn PA, Donnelly LM, Calder DA, et al. The Saskatchewan Surgical Care Network — toward timely and appropriate access. Hosp Q 2003;7:44-8.

17. Arnett G, Hadorn DC; Steering Committee of the Western Canada Waiting List Project. Developing priority criteria for hip and knee replacement: results from the Western Canada Waiting List Project. Can J Surg 2003;46:290-6.

18. Conner-Spady B, Estey A, Arnett G, et al. Prioritization of patients on waiting lists for hip and knee replacement: validation of a priority criteria tool. Int J Technol Assess Health Care 2004;20:509-15.

19. Glass GV, Hopkins KD. Statistical methods in education and psychology. 3rd ed. Needham Heights (MA): Allyn and Bacon; 1996.

20. Conner-Spady BL, Johnston GH, Sanmartin C, et al. A bird can't fly on one wing: patient views on waiting for hip and knee replacement surgery. Health Expect 2007;10:108-16.

21. Brooks R. EuroQol: the current state of play. Health Policy 1996;37:53-72.

22. Ostendorf M, van Stel HF, Buskens E, et al. Patient-reported outcome in total hip replacement. A comparison of five instruments of health status. J Bone Joint Surg Br 2004;86:801-8.

23. Hosmer DW, Lemeshow S. Applied logistic regression. New York (NY): John Wiley \& Sons; 1989.

24. Brunenberg DE, van Steyn MJ, Sluimer JC, et al. Joint recovery programme versus usual care: an economic evaluation of a clinical pathway for joint replacement surgery. Med Care 2005;43:1018-26.

25. Magee H, Davis LJ, Coulter A. Public views on healthcare performance indicators and patient choice. J R Soc Med 2003;96:338-42.

26. Coulter A, Jenkinson C. European patients' views on the responsiveness of health systems and healthcare providers. Eur J Public Health 2005;15:355-60.

27. Marshall MN, Shekelle PG, Leatherman S, et al. The public release of performance data: What do we expect to gain? A review of the evidence. JAMA 2000;283:1866-74.

28. Schneider EC, Epstein AM. Use of public performance reports: a survey of patients undergoing cardiac surgery. JAMA 1998;279:1638-42.

Correspondence to: Dr. Barbara Conner-Spady, University of Calgary, Heritage Medical Research Building, 3330 Hospital Dr. $N W$, Calgary AB T2N 4N1; barbara.spady@ualberta.net 